\title{
SOME PROPERTIES OF CLOSED 1-FORMS ON A SPECIAL RIEMANNIAN MANIFOLD
}

\author{
GR. TSAGAS
}

\begin{abstract}
Let $M$ be a compact Riemannian manifold whose sectional curvature is strictly negative; then every closed 1 -form on $M$ has a singularity.
\end{abstract}

1. Introduction. Let $M$ be a compact Riemannian manifold. If we assume that the dimension of the manifold is even and the sectional curvature of $M$ is negative $\delta$-pinched, where $\delta>\left(n^{2}+5 n+7\right) /\left(n^{2}+6 n+14\right)$, then every harmonic 1-form on $M$ has a singularity [9], where $n=\operatorname{dim} M$.

The main purpose of the present paper is to generalize the above result. Now this result can be stated as follows. Let $M$ be a compact Riemannian manifold whose sectional curvature is strictly negative; then every closed 1-form on $M$ has a singularity.

2. We consider a compact Riemannian manifold $M$, whose sectional curvature is strictly negative. We assume that there is a closed 1 -form $w$ on $M$ without singularities. Then from [10, p. 153] we obtain that the manifold is the total space of a fibre bundle whose base manifold is a circle, that is

$$
F \stackrel{i}{\rightarrow} M \stackrel{p}{\rightarrow} S .
$$

From this fibre bundle we have the following exact sequence [5, p. 153]:

$$
\begin{aligned}
\cdots \stackrel{p_{*}}{\rightarrow} \pi_{n+1}\left(S, b_{0}\right) \stackrel{d_{*}}{\rightarrow} \pi_{n}\left(F, e_{0}\right) \stackrel{i_{*}}{\rightarrow} \pi_{n}\left(M, e_{0}\right) \stackrel{p_{*}}{\rightarrow} \pi_{n}\left(S, b_{0}\right) \\
\rightarrow \cdots \stackrel{p_{*}}{\rightarrow} \pi_{1}\left(S, b_{0}\right) \stackrel{d_{*}}{\rightarrow} \pi_{0}\left(F, e_{0}\right) \stackrel{i_{*}}{\rightarrow} \pi_{0}(M, e),
\end{aligned}
$$

where $b_{0} \in S$ is a basic point and the fibre $F=p^{-1}\left(b_{0}\right)$ is not empty, which is called a basic fibre and choose $e_{0} \in F$ as a basic point.

From the above exact sequence we obtain

$$
\pi_{2}\left(S, b_{0}\right) \stackrel{d_{*}}{\rightarrow} \pi_{1}(F, e) \stackrel{i_{*}}{\rightarrow} \pi_{1}\left(M, e_{0}\right) \stackrel{p_{*}}{\rightarrow} \pi_{1}\left(S, b_{0}\right) \stackrel{d_{*}}{\rightarrow} \pi_{0}\left(F, e_{0}\right)
$$

Since $\pi_{1}\left(S, b_{0}\right)=\mathbf{Z}, \pi_{2}\left(S, b_{0}\right)=0$ and we can assume $\pi_{0}(F, e)=0$, [3], then the sequence (2.2) takes the form:

$$
0 \stackrel{d_{*}}{\rightarrow} \pi_{1}(F, e) \stackrel{i_{*}}{\rightarrow} \pi_{1}\left(M, e_{0}\right) \stackrel{p_{*}}{\rightarrow} \mathrm{Z} \rightarrow 0 .
$$

From the exact sequence (2.3) we conclude that the mapping $i_{*}$ is injective and the mapping $p_{*}$ is surjective.

Received by the editors March 6, 1979 and, in revised form, November 16, 1979.

1980 Mathematics Subject Classification. Primary 53C20.

Key words and phrases. Riemannian manifold, closed 1-form, harmonic 1-form, negative $\delta$-pinched, singularity of 1-forms. 
The exact sequence (2.3) can be written

$$
G_{1} \stackrel{f_{1}}{\rightarrow} G_{2} \stackrel{f_{2}}{\rightarrow} G_{3}
$$

where $G_{1}=\pi_{1}(F, e), G_{2}=\pi_{1}\left(M, e_{0}\right), G_{3}=\mathbf{Z}, i_{*}=f_{1}$ and $p_{*}=f_{2}$.

We assume that $F$ is not simply connected. That means $G_{1}=\pi_{1}(F, e)$ is not trivial.

From the exact sequence (2.4) and since $f_{1}$ is injective and $f_{2}$ surjective we can consider that $G_{1}$ is a normal subgroup of $G_{2}$ and the quotient group $G_{2} / G_{1}$ is isomorphic to $G_{3}$.

Since $G_{3}$ is an infinite cycle we obtain that $G_{2}$ considered as a set has the form

$$
\left(\ldots, a^{-2} G_{1}, a^{-1} G_{1}, G_{1}, a G_{1}, a^{2} G_{1}, \ldots\right),
$$

or the form

$$
\left(\ldots, G_{1} a^{-2}, G_{1} a^{-1}, G_{1}, G_{1} a, G_{1} a^{2}, \ldots\right),
$$

where $a$ is an element of the group $G_{2}$.

Since $G_{1}$ is a normal subgroup of $G_{2}$ we have $a^{-1} G_{1} a=G_{1}$; that is, there is an element $a$ of $G_{2}$ such that $a^{-1} G_{1} a \cap G_{1}=G_{1} \supset\{e\}$ where $e$ is the identity element of $G_{2}$.

The following proposition is known [1, p. 46].

Proposition (2.1). Let $M$ be a compact Riemannian manifold whose sectional curvature is strictly negative. If $G=\pi_{1}(M, m, \gamma)$ is a ray subgroup of $\pi_{1}(M, m)$ and $\beta \notin G$, then $\beta G \beta^{-1} \cap G=\{e\}$, where $e$ is the identity element of $\pi_{1}(M, m)$.

From the above and Proposition (2.1) we have a contradiction. We have reached a contradiction because we have assumed that the Riemannian manifold $M$ admits a closed 1-form without singularities. If $F$ is simply connected, then $\pi_{1}(F, e)$ is trivial and $G_{2}=\pi_{1}\left(M, e_{0}\right)=\mathbf{Z}$, which is not true for a compact manifold with negative sectional curvature [7].

Therefore we obtain the theorem.

THEOREM (2.1). Let $M$ be a compact Riemannian manifold whose sectional curvature is strictly negative. Then every closed 1-form on $M$ has at least one singularity.

From this theorem we have the corollary.

COROllary (2.1). Let $M$ be a compact Riemannian manifold whose sectional curvature is strictly negative. Then every harmonic 1-form on $M$ has at least one singularity.

\section{REFERENCES}

1. R. L. Bishop and B. O'Neil, Manifolds of negative curvature, Trans. Amer. Math. Soc. 145 (1969), $1-49$.

2. W. Byers, On a theorem of Preissmann, Proc. Amer. Math. Soc. 24 (1970), 50-51.

3. F. T. Farrell, The obstruction to fibering a manifold over a circle, Indiana Univ. Math. J. 21 (1971), 315-346.

4. D. Gromol and J. Wolf, Some relations between the metric structure and the algebraic structure of the fundamental group in the manifolds of nompositive curvature, Bull. Amer. Math. Soc. 77 (1971), 545-552. 
5. S. T. Hu, Homotopy theory, Academic Press, New York, 1963.

6. J. Milnor, Lectures on Morse theory, Ann. of Math. Studies, No. 51, Princeton Univ. Press, Princeton, N.J., 1963.

7.

8. __ Growth of finitely generated solvable groups, J. Differential Geometry 2 (1968), 427-449.

9. Gr. Tsagas, On the singularities of harmonic 1-forms on a Riemannian manifold, Kōdai Math. Sem. Rep. 26 (1975), 459-466.

10. D. Tischler, On fibering certain foliated manifolds over $S^{1}$, Topology 9 (1970), 153-154.

11. J. A. Wolf, Growth of finitely generated solvable groups and curvature of Riemannian manifolds, J. Differential Geometry 2 (1970), 421-446.

12. S. Yau, On the fundamental group of compact manifolds of non-positive curvature, J. Differential Geometry 6 (1971), 579-585.

Department of Mathematics, Faculty of Technology, University of Thessaloniki, ThessaLONIKI, GREECE 